\title{
Circular Antenna Array Synthesis Using Multiverse Optimizer
}

\author{
Ahmet Emre Taser $\mathbb{D}^{1},{ }^{1}$ Kerim Guney, ${ }^{2}$ and Erhan Kurt ${ }^{2}$ \\ ${ }^{1}$ Graduate School of Natural and Applied Sciences, Erciyes University, Kayseri 38039, Turkey \\ ${ }^{2}$ Department of Electrical and Electronics Engineering, Nuh Naci Yazgan University, Kayseri 38170, Turkey \\ Correspondence should be addressed to Ahmet Emre Taser; aetaser@gmail.com
}

Received 6 March 2020; Accepted 25 July 2020; Published 24 August 2020

Academic Editor: Symeon Nikolaou

Copyright $\odot 2020$ Ahmet Emre Taser et al. This is an open access article distributed under the Creative Commons Attribution License, which permits unrestricted use, distribution, and reproduction in any medium, provided the original work is properly cited.

\begin{abstract}
Antenna array synthesis is one of the most popular topics in the electromagnetic field. Since achieving a desired antenna radiation pattern is a mathematical problem, in the literature, there are various optimization algorithms applied to the synthesis process of different kinds of antenna arrays. In this study, Multiverse Optimizer (MVO) and modified MVO (MMVO) are used to perform circular antenna array (CAA) synthesis. During the exploration, exploitation, and local search phases of calculation, MVO uses three concepts in cosmology; white hole, black hole, and wormhole. Convergence capability of this nature-inspired algorithm is employed for finding optimum amplitude and position values of CAA elements in order to achieve an array pattern with low maximum sidelobe level (MSL) and minimum circumference. The performance of MVO and MMVO was tested on five design examples of pattern synthesis, and the obtained results were compared with ten different algorithms. The simulation results show that MVO and MMVO provide low MSLs with small circumferences.
\end{abstract}

\section{Introduction}

In recent days, with the developing technology, devices have started to host more wireless components. The application of wireless technology in all areas of data transfer and communication has accelerated the spread of its use. However, increment in use of this technology brought some drawbacks such as interference and electromagnetic pollution. To overcome this issue and use frequency spectrum much more efficiently, RF components are improved year by year. Antennas, which constitute one of the most important elements of wireless technology, also took part in this improvement. As a result, different antenna types appeared such as wire, horn, dish, and array.

The antenna arrays consist of a combination of multiple antenna elements and they are widely used in communication. Electromagnetic radiation of each element can be constructively or destructively added. Thus, whole array can radiate towards desired direction while minimizing the interference on undesired angles [1]. Thanks to their useful and flexible structure, antenna arrays can provide high gain, high directivity, and high beam steering capability with less interfering effect. These features of antenna arrays make them popular both in military and civil applications. They are widely used in sonar, radar, navigation systems, cellular systems, and satellite communications.

Antenna arrays take their names according to the geometric shape of overall array structure. Array elements can be positioned on a single line, called linear antenna arrays, or distributed over a plane, called planar antenna arrays. Array structures can be in form of circle and ellipse too. Moreover, by creating nested structures from circular or elliptical arrays, concentric circular and concentric elliptical antenna arrays are achieved.

In this paper, circular antenna array (CAA) is considered for the array synthesis. Unlike the linear antenna arrays, the main lobe of CAA can be directed between 0 and 360 degrees. According to the changes in position, amplitude, and phase of array elements, or changes in circle radius, overall radiation pattern is shaped. However, achieving a desired antenna pattern is a challenging task since the mentioned array parameters have to be calculated. It is seen from the literature that the optimization algorithms can be used to overcome this kind of pattern synthesis problems. The 
following can be given as examples of these studies: linear antenna array synthesis using Tabu Search Algorithm (TSA) [2], Harmony Search Algorithm (HSA) [3], and Touring Ant Colony Optimization (TACO) [4]; planar antenna array synthesis using Taguchi Method (TM) [5] and Genetic Algorithm (GA) [6]; elliptical antenna array synthesis using Backtracking Search Optimization Algorithm (BSA) [7]; circular antenna array synthesis using Differential Evolution (DE) Algorithm [8]; and concentric CAA synthesis using BSA [9].

Multiverse Optimizer (MVO), which is a nature-inspired algorithm, was proposed in 2016 [10] and used to solve practical optimization problems in engineering such as welded beam design, gear train design, three-bar truss design, pressure vessel design, and cantilever beam design. In [10], the performance of MVO was compared with GA, Particle Swarm Optimization (PSO), Grey Wolf Optimizer (GWO), and Gravitational Search Algorithm (GSA). The majority of test results show that MVO outperforms the popular algorithms in the literature. MVO was also used for multiobjective optimal antenna synthesis of microwave wireless power transmission [11].

The main objective of this paper is to compute optimally the amplitudes and positions of CAA elements to achieve low MSL and small circumference using MVO and modified MVO (MMVO). Synthesis process was applied for CAAs with 8, 10, 12 , and 20 elements. The obtained results were compared with the results of ten different optimization algorithms: hybrid algorithm (HA) [12] based on Antlion Optimization (ALO) and Grasshopper Optimization Algorithm (GOA), ALO [12], Sequential Quadratic Programming (SQP) [13], Self-Adaptive Differential Evolution (SADE) [13], Biogeography Based Optimization (BBO) [13], GA [14], PSO [15], Firefly Algorithm (FA) [16], Seeker Optimization Algorithm (SOA) [17], and Simulated Annealing (SA) [18].

In the following section, MVO and MMVO are explained in detail. Section 3 includes the CAA geometry and equation of array factor. Section 4 presents results and discussions. Section 5 covers the conclusion part.

\section{Multiverse Optimizer}

Multiverse theory was proposed by physicists, and MVO is inspired by this theory. It is believed that more than one big bang occurred, and each one of them is resulted with the birth of a universe $[19,20]$. The idea behind this theory is the opposite of universe, which states the presence of other universes in addition to ours. Besides, according to this model, these universes may interact and/or collide with each other.

MVO is based on three concepts in the cosmology: white holes, black holes, and wormholes [10]. Physicists state that the white hole, which is also considered as big bang, is the main component of the birth of a universe [21]. It appeared after the collision of two parallel universes [22]. Black holes are the white holes in reverse and, because of their extremely high gravitational force, they attract everything including light beams [23, 24]. Wormholes can be described as time/ space highways where objects are able to travel instantly between two different points of a universe or two different universes too [25].

Basically, MVO converges the target by sending and receiving the objects (variables) between the universes (solutions). This sending/receiving process depends on the inflation rates (fitness values) of the universes calculated over iterations. If a universe has high inflation rate, it is highly probable to have white holes and tends to send object towards other universes. If a universe has low inflation rate, most probably it has a black hole and tends to receive objects. This mentioned process is the exploration step of the algorithm and is represented mathematically as follows [10]:

$$
\begin{aligned}
& U=\left[\begin{array}{cccc}
x_{1}^{1} & x_{1}^{2} & \cdots & x_{1}^{p} \\
x_{2}^{1} & x_{2}^{2} & \cdots & x_{2}^{p} \\
\vdots & \vdots & \vdots & \vdots \\
x_{n}^{1} & x_{n}^{2} & \cdots & x_{n}^{p}
\end{array}\right], \\
& x_{i}^{j}=\left\{\begin{array}{ccc}
x_{k}^{j}, & r 1<\mathrm{NI}(U i), \\
x_{i}^{j}, & \text { otherwise },
\end{array}\right.
\end{aligned}
$$

where $U i$ is the $i$ th universe, $p$ is the number of parameters (variables), $n$ is the number of universes (solutions), $x_{i}^{j}$ is the $j$ th parameter of $i$ th universe, $r 1$ is a random number in $[0$, $1]$, NI (Ui) is normalized inflation rate of the $i$ th universe, and $x_{k}^{j}$ is the $j$ th parameter of $k$ th universe selected by a roulette wheel selection mechanism.

In the exploitation phase of the algorithm, it is assumed that each universe is using wormholes to transfer the objects through space randomly regardless of inflation rate. Besides, wormholes are used to perform local updates in each universe by establishing a connection to the best universe formed so far. This mechanism is formulated as follows [10]:

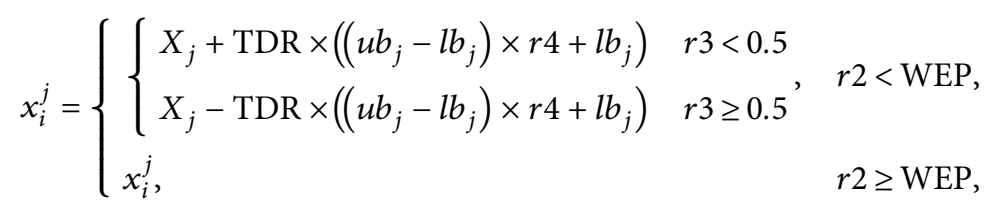

where $X_{j}$ is the $j$ th parameter of best universe formed so far; $r 2$, $r 3$, and $r 4$ are the random numbers in $[0,1]$; and $\mathrm{ub}_{j}$ and $\mathrm{lb}_{j}$ are used to define upper and lower bound of $j$ th variable, respectively. WEP and TDR are coefficients which are explained below.
WEP stands for the wormhole's existence probability in the universe. In the algorithm, this coefficient is increased linearly over repetitions to emphasize exploitation. WEP is calculated as follows [10]: 


$$
\mathrm{WEP}=\min +l \times\left(\frac{\max -\min }{L}\right),
$$

where, by default, min is defined as 0.2 and max is defined as 1. $L$ indicates the maximum iteration value and $l$ represents the current step of the iteration.

Traveling distance rate (TDR) is also a coefficient. It is used while sending objects through a wormhole to the best universe obtained so far. The formula for TDR is as follows [10]:

$$
\operatorname{TDR}=1-\frac{l^{(1 / p)}}{L^{(1 / p)}}
$$

where $p$ indicates the exploitation accuracy and is equal to 6 as a default value.

The pseudocode of the MVO is shown in Figure 1.

2.1. Modified MVO (MMVO). As is stated in No Free Lunch (NFL) Theorem, there is no single algorithm to solve every problem set [26]. Besides, it is realized that the performance of an algorithm can be improved by modifying it and thus the algorithm can be used to solve problems in different fields. Even though the performance of original MVO is good enough to optimize the problems, structure of the algorithm itself is suitable for modifications to improve the convergence. Therefore, in this paper, the exploitation phase of the MVO is changed as follows:

$$
x_{i}^{j}=\left\{\begin{array}{lll}
\left\{X_{j}+\mathrm{TDR} \times\left(\left(X_{j}-x_{i}^{j}\right) \times r 4+x_{i}^{j}\right)\right. & r 3<0.5 & \\
X_{j}-\mathrm{TDR} \times\left(\left(X_{j}-x_{i}^{j}\right) \times r 4+x_{i}^{j}\right) & r 3 \geq 0.5 & r 2<\mathrm{WEP}, \\
x_{i}^{j}, & & r 2 \geq \mathrm{WEP},
\end{array}\right.
$$

and this modification aims to perform the solution updates locally using the best universe and current universe instead of upper and lower bounds of problem set. Thus, by shortening the exploitation steps, the algorithm is improved and forced to find better amplitude and position values for array elements.

\section{Circular Antenna Array}

In Figure 2, geometry of a nonuniform CAA is depicted. $N$ isotropic radiators (antenna elements) are placed nonuniformly on a circle of radius $a$ in the $x-y$ plane. The array factor of such structure can be mathematically described as follows [1]:

$$
\operatorname{AF}(\theta, \phi)=\sum_{n=1}^{N} I_{n} \exp \left(j\left[k a \sin (\theta) \cos \left(\phi-\phi_{n}\right)+\alpha_{n}\right]\right),
$$

where

$$
\begin{aligned}
& k a=\frac{2 \pi}{\lambda} a=\sum_{i=1}^{N} d_{i}, \\
& \phi_{n}=\frac{2 \pi \sum_{i=1}^{n} d_{i}}{k a} .
\end{aligned}
$$

In order to focus the main beam towards the $\left(\theta_{o}, \phi_{o}\right)$ direction, the excitation phase is written as follows [1]:
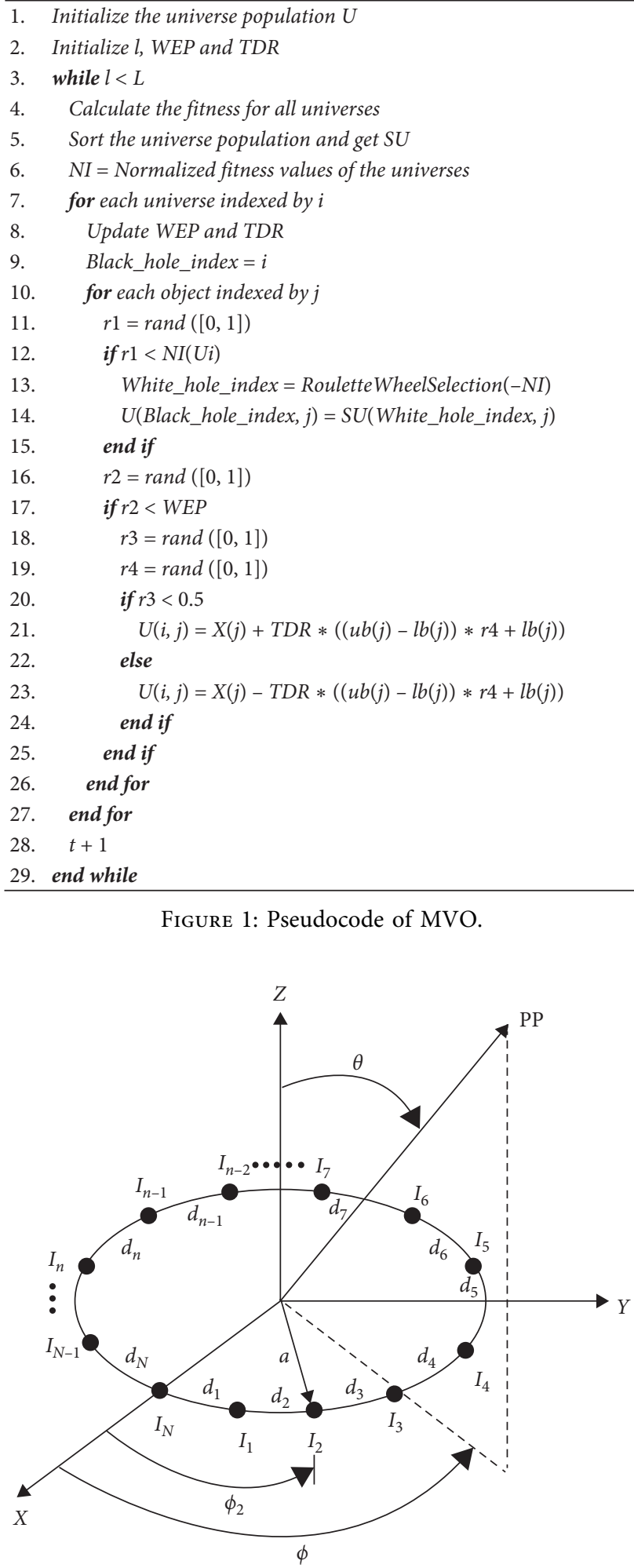

Figure 2: Geometry of a nonuniform CAA.

$$
\alpha_{n}=-k a \sin \left(\theta_{o}\right) \cos \left(\phi_{o}-\phi_{n}\right) .
$$

In the above, $I_{n}$ and $\alpha_{n}$ indicate the excitation amplitude and excitation phase of the $n$th antenna element, respectively. $d$ is used to define the arc distance between the adjacent antenna elements. $\phi_{n}$ is the angular position of the $n$th radiator on $x-y$ 


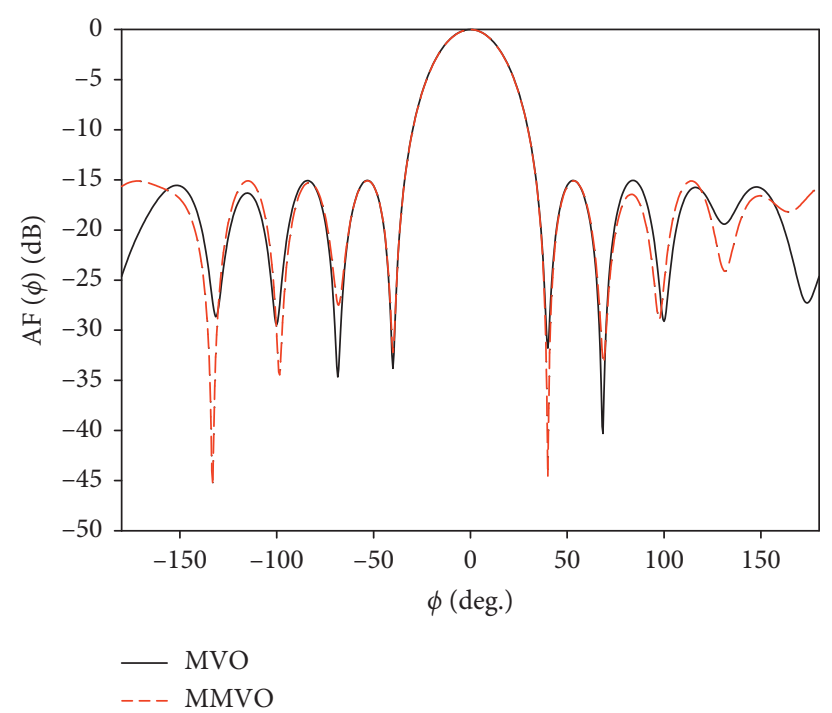

Figure 3: Radiation pattern of the optimized 8-element CAA.

plane. $\theta$ represents the measured elevation angle from the positive $z$-axis, and $\phi$ is used to represent the measured azimuth angle from the positive $x$-axis. Since the antenna array is placed in the $x-y$ plane, $\theta$ is assumed to be $90^{\circ}$. $\left(\theta_{o}, \phi_{o}\right)$ shows the direction of the main beam of antenna array. In order to simplify the calculations, $\theta_{o}$ is taken as $90^{\circ}$ and $\phi_{o}$ is taken as $0^{\circ}$. In other words, the main beam is focused along the positive $x$-axis.

\section{Results and Discussion}

In this section, the overall performance of $\mathrm{MVO}$ and MMVO on CAA synthesis is evaluated in detail. The purpose of performing array synthesis is to achieve a desired antenna pattern. As mentioned in the Introduction, different synthesis processes were applied to different kinds of antenna arrays with different algorithms. The main aim of those works is to get low sidelobe levels, place nulls in desired directions, and increase the gain or directivity of the antenna.

In this paper, MVO and MMVO algorithms were used to find optimum amplitude and position values of CAA elements in order to achieve low MSL and minimum circumference. The obtained results from both MVO and MMVO were comprehensively compared with the results of ten different algorithms which are GA [14], PSO [15], FA [16], SQP [13], SADE [13], HA [12], ALO [12], BBO [13], SOA [17], and SA [18]. During the optimization, the following fitness function was used:

$$
\text { Fitness }=\max \left(20 \log \left|\frac{\mathrm{AF}\left(\phi_{\mathrm{MSL}}\right)}{\mathrm{AF}_{\text {max }}}\right|\right)+\left|\left(\left(\sum_{i=1}^{N} d_{i}\right)-(C)\right)\right| \text {, }
$$

where $\phi_{\mathrm{MSL}}$ is the angular interval of the sidelobe region that is minimized, $\mathrm{AF}_{\max }$ is the peak of main beam, and $C$ is the desired circumference. In each iteration step, the difference between the desired circumference and the arc distance summation is taken into account and the algorithm reduces it while keeping the sidelobe level to a minimum. The fitness function is used for CAA with $8,10,12$, and 20 elements. In order to observe the real performance of MVO and MMVO and to make a fair comparison, 20 independent runs were performed under the default parameters. All simulations were done by setting the algorithm to 300 iterations with population size of 50 on MATLAB R2016b with a computer that has $2.4 \mathrm{GHz}$ i7 processor and $8 \mathrm{~GB}$ RAM.

In the first example, the optimum amplitude and position values of CAA with 8 elements are calculated by MVO and MMVO to get the minimum sidelobe level in the region $\phi=\left[-180^{\circ},-34^{\circ}\right]$ and $\phi=\left[34^{\circ}, 180^{\circ}\right]$. The resultant radiation patterns of MVO and MMVO are shown in Figure 3. Table 1 illustrates MSL and circumference values of the radiation patterns obtained using MVO and MMVO. Table 1 also shows the comparison results of GA [14], PSO [15], FA [16], SQP [13], SADE [13], HA [12], ALO [12], BBO [13], SOA [17], and SA [18]. It can be clearly observed from Table 1 that MSL and circumference values of MVO and MMVO are better than those of GA [14], PSO [15], FA [16], SQP [13], SADE [13], HA [12], ALO [12], BBO [13], SOA [17], and SA [18]. The convergence curves of this example can be seen in Figure 4 .

In the second example, the number of array elements is increased from 8 to 10 and the sidelobe region is taken as $\phi=\left[-180^{\circ},-27^{\circ}\right]$ and $\phi=\left[27^{\circ}, 180^{\circ}\right]$. The algorithm has been run 20 times. The patterns of this design example can be seen in Figure 5. The MSL and circumference results of MVO and MMVO are listed in Table 1 in comparison with GA [14], PSO [15], FA [16], SQP [13], SADE [13], HA [12], ALO [12], BBO [13], SOA [17], and SA [18]. As can be seen from this table, the MSL and circumference values achieved by MVO and MMVO are better than those by the other compared algorithms.

The 12-element CAA is the third design example of this part. The best MSL and circumference results are obtained after 20 runs in the $\phi=\left[-180^{\circ},-23^{\circ}\right]$ and $\phi=\left[23^{\circ}, 180^{\circ}\right]$ angular region. The radiation patterns are given in Figure 6. In Table 1, the MSL and circumference values of MVO and MMVO are compared with those of GA [14], PSO [15], FA 


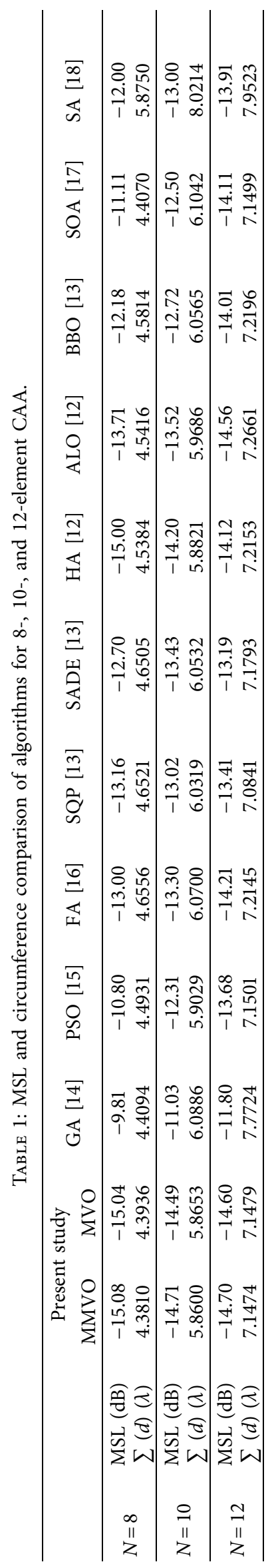




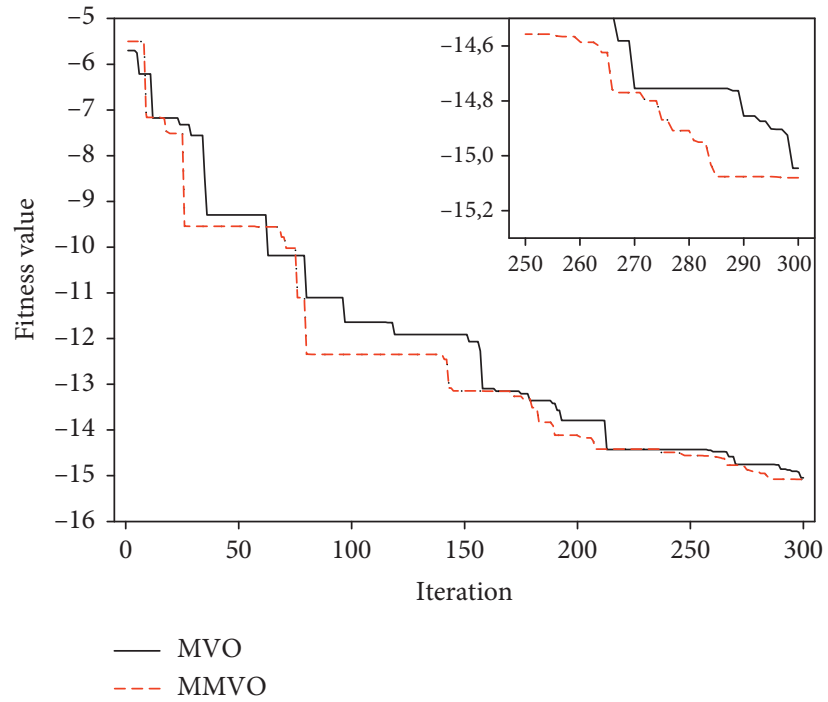

Figure 4: Convergence curve of 8-element CAA.

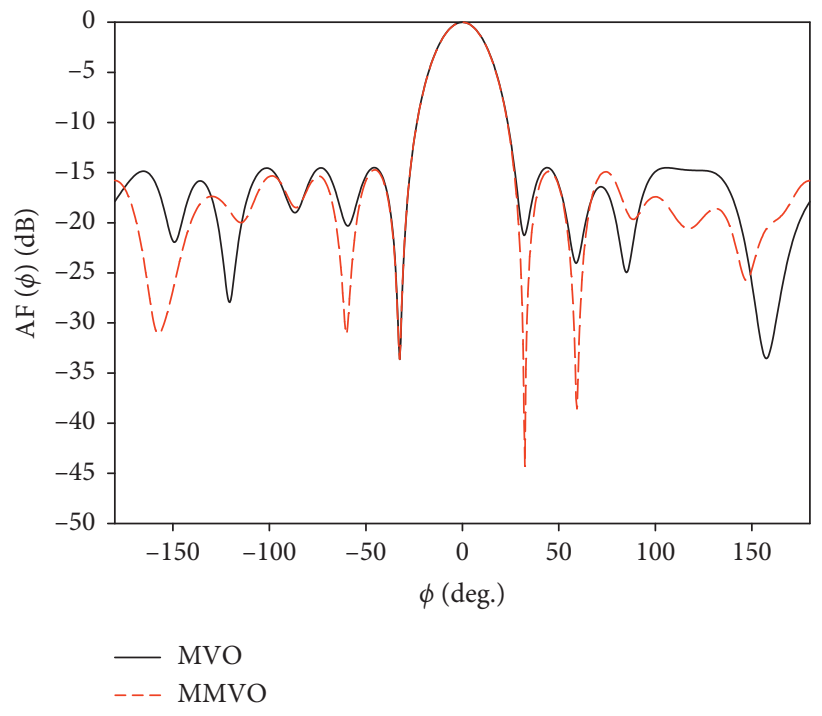

FIGURE 5: Radiation pattern of the optimized 10-element CAA.

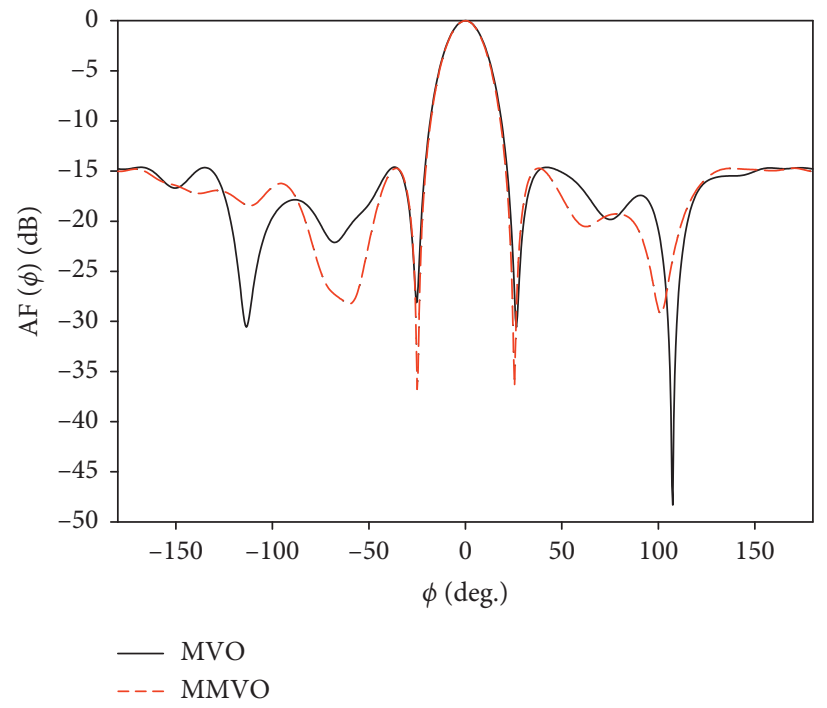

FIGURE 6: Radiation pattern of the optimized 12-element CAA. 


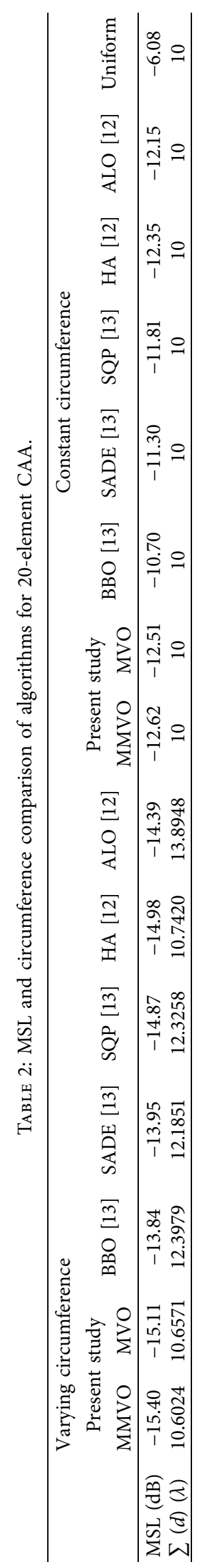




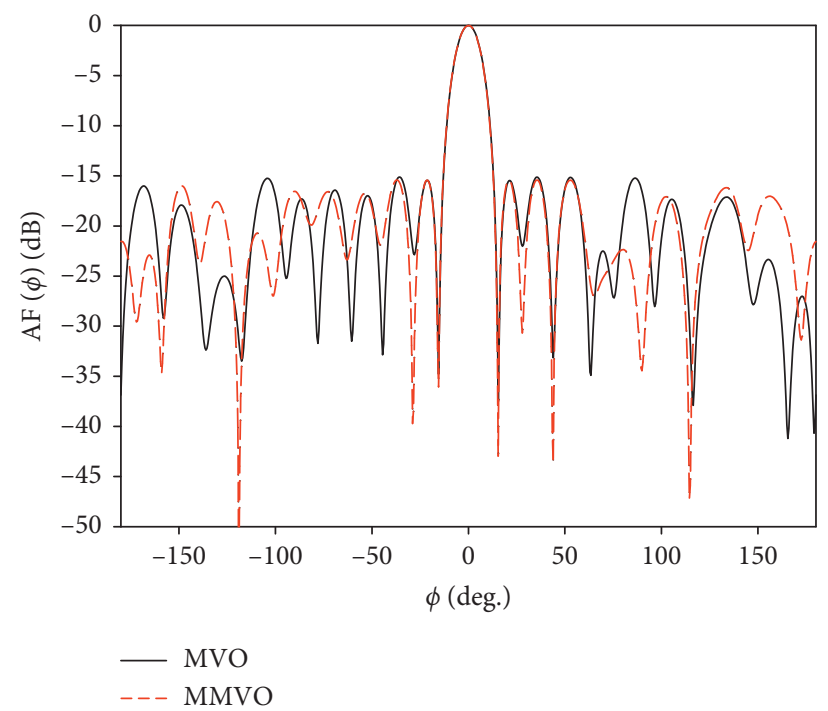

Figure 7: Radiation pattern of the optimized 20-element CAA.

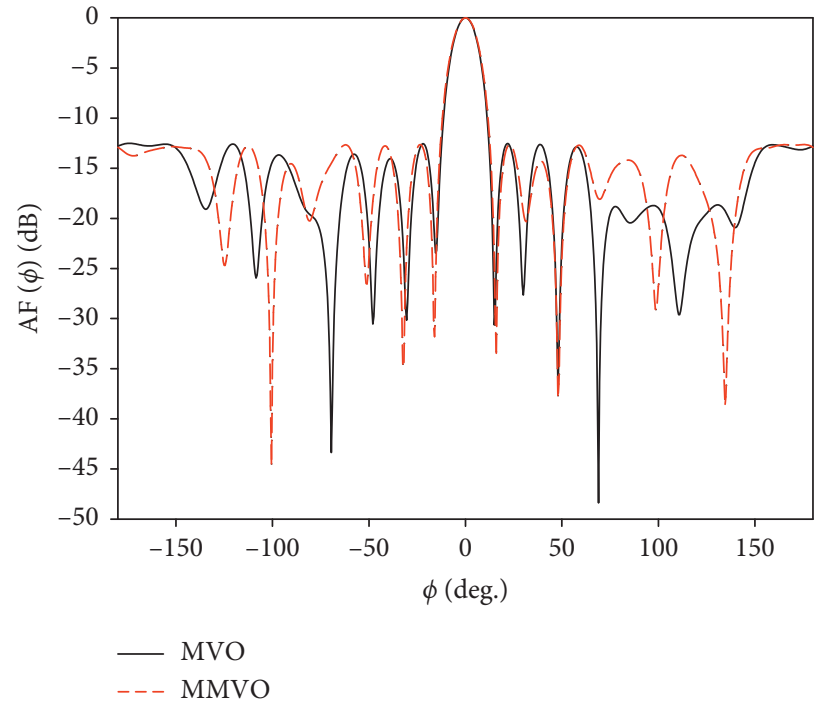

Figure 8: Radiation pattern of the optimized 20-element CAA with $10 \lambda$ circumference.

TABLe 3: Amplitude and position values of array elements for design examples.

\begin{tabular}{ccc}
\hline & & {$\left[d_{1}, d_{2}, d_{3}, \ldots, d_{\mathrm{N}}\right]$ in $\lambda^{\prime} s$} \\
{$\left[I_{1}, I_{2}, I_{3}, \ldots, I_{N}\right]$}
\end{tabular}


TABle 3: Continued.

\begin{tabular}{|c|c|c|}
\hline & & $\begin{array}{c}{\left[d_{1}, d_{2}, d_{3}, \ldots, d_{\mathrm{N}}\right] \text { in } \lambda^{\prime} \mathrm{s}} \\
{\left[I_{1}, I_{2}, I_{3}, \ldots, I_{N}\right]}\end{array}$ \\
\hline \multirow{2}{*}{ Figure 7} & MVO & $\begin{array}{c}0.2479,0.4334,0.9496,0.7325,0.6853,0.5666,0.9143,0.5344,0.1350,0.3199,0.1926,0.4758,0.7640,0.2852,0.4629, \\
0.7318,0.5492,0.9961,0.5618,0.1188 \\
0.5082,0.7995,0.7677,0.6978,0.5223,0.7467,0.6694,0.7362,0.7616,0.8147,0.6043,0.3239,0.7624,0.2968,1,0.8843, \\
0.9138,0.6642,0.9445,0.8043\end{array}$ \\
\hline & MMVO & $\begin{array}{c}0.3013,0.4719,0.8251,0.1155,0.6402,0.8880,0.3553,0.9541,0.5113,0.1000,0.2801,0.4103,0.8738,0.2270,0.6347 \\
0.7450,0.6127,0.9866,0.4472,0.2223 \\
0.7599,0.2832,0.1555,0.6541,0.3102,0.5869,0.9399,0.3185,1,0.1036,0.5827,0.7808,0.1381,0.4406,0.6412,0.7885 \\
0.5329,0.5145,0.9146,0.4414\end{array}$ \\
\hline \multirow{2}{*}{ Figure 8} & MVO & $\begin{array}{c}0.1008,0.1130,0.4527,0.4436,0.9314,0.2575,0.2372,0.3995,0.8275,0.9908,0.3814,1.0813,0.9903,0.3910,0.3395, \\
0.8914,0.4817,0.4714,0.1111,0.1069 \\
0.5859,0.5800,0.9237,0.6956,0.7435,0.8154,0.3572,0.7263,0.9135,0.7137,0.9515,0.7478,0.7961,0.7528,1,0.6600, \\
0.9821,0.3981,0.7731,0.5785\end{array}$ \\
\hline & MMVO & $\begin{array}{c}0.6364,0.3793,0.9334,0.5274,0.3513,0.9207,0.4730,0.1859,0.5125,0.3305,0.5517,0.3355,0.8594,0.5929,0.3201, \\
0.6699,0.2335,0.5339,0.3883,0.2644 \\
0.7025,0.7391,0.2558,0.3866,0.8487,0.5303,0.2365,0.7463,1,0.8262,0.1349,0.9108,0.1730,0.7486,0.6675,0.2566, \\
0.7451,0.2617,0.6425,0.6143\end{array}$ \\
\hline
\end{tabular}

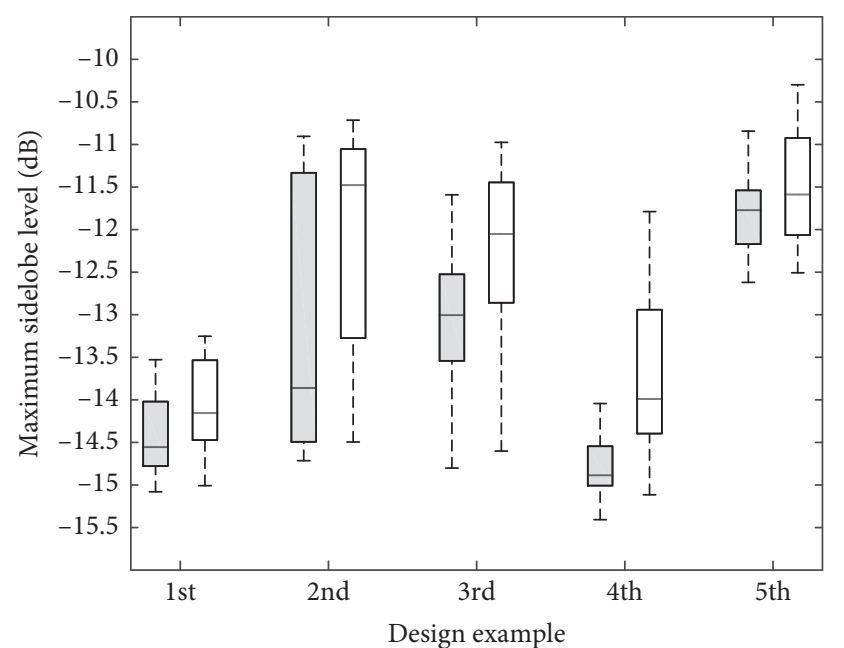

MVO

MMVO

Figure 9: Box-and-whisker plots of design examples 1, 2, 3, 4, and 5 for 20 runs.

[16], SQP [13], SADE [13], HA [12], ALO [12], BBO [13], SOA [17], and SA [18]. It is clearly seen from Table 1 that MSL and circumference values of the methods proposed in this study are better than those of the other algorithms except that the circumference value of SQP [13] is slightly better than that of our methods.

In the fourth example, the number of array elements is set to 20 and the sidelobe suppression region is expanded to $\phi=\left[-180^{\circ},-14^{\circ}\right]$ and $\phi=\left[14^{\circ}, 180^{\circ}\right]$. Result comparison of this synthesis example is made in Table 2, and array patterns are shown in Figure 7. According to Table 2, it is evidently seen that the MSL and circumference values obtained by MVO and MMVO are better than those of BBO [13], SADE [13], SQP [13], HA [12], and ALO [12].

In the last example, while suppressing the MSL, the circumference value is fixed to the circumference value of 20-element uniform CAA which is $10 \lambda\left(d_{i}=0.5 \lambda\right)$. After 20 independent runs, $-12.51 \mathrm{~dB}$ and $-12.62 \mathrm{~dB}$ MSL are achieved for MVO and MMVO, respectively. The obtained patterns are shown in Figure 8. In Table 2, the MSL results of patterns produced by MVO and MMVO are compared with the results of BBO [13], SADE [13], SQP [13], HA [12], and ALO [12]. It is apparent from Table 2 that MSL values of MVO and MMVO are better than the MSL values of the other compared algorithms.

The amplitude and position values for all design examples are shown in Table 3. Besides, box-and-whisker plots are depicted in Figure 9 to exhibit the overall statistical view of 20 independent runs for each design case. From this figure, improvement in results can be seen as an outcome of modification in the algorithm.

\section{Conclusions}

In this paper, MVO and MMVO algorithms were presented for the synthesis of CAA. These algorithms were used to calculate the optimum amplitude and position values of the isotropic radiators in the CAA in order to achieve low MSL and minimum circumference. Five different design examples were used to see the overall performance of the MVO and MMVO against ten different optimization algorithms. It was observed that MVO and MMVO have offered minimum sidelobe levels with competitive circumference values. MVO and MMVO can be a good alternative while solving the optimization problems in antenna and electromagnetics.

\section{Data Availability}

The data used to support the findings of this study are included within the article.

\section{Conflicts of Interest}

The authors declare that there are no conflicts of interest regarding the publication of this paper. 


\section{References}

[1] C. A. Balanis, Antenna Theory: Analysis and Design, WileyInterscience, Hoboken, NJ, USA, 3rd edition, 2005.

[2] A. Akdagli and K. Guney, "Null steering of linear antenna arrays by phase perturbations using modified tabu search algorithm," Journal of Communications Technology and Electronics, vol. 49, no. 1, pp. 37-42, 2004.

[3] K. Guney and M. Onay, "Optimal synthesis of linear antenna arrays using a harmony search algorithm," Expert Systems with Applications, vol. 38, no. 12, pp. 15455-15462, 2011.

[4] A. A. Akdagli, K. Guney, and D. Karaboga, "Touring ant colony optimization algorithm for shaped-beam pattern synthesis of linear antenna," Electromagnetics, vol. 26, no. 8, pp. 615-628, 2006.

[5] A. Smida, R. Ghayoula, A. Ferchichi, A. Gharsallah, and D. Grenier, "Planar antenna array pattern synthesis using a taguchi optimization method," International Journal on Communications Antenna and Propagation, vol. 3, no. 3, pp. 158-162, 2013.

[6] D. Marcano and F. Duran, "Synthesis of antenna arrays using genetic algorithms," IEEE Antennas and Propagation Magazine, vol. 42, no. 3, pp. 12-20, 2000.

[7] K. Guney and A. Durmus, "Elliptical antenna array synthesis using backtracking search optimisation algorithm," Defence Science Journal, vol. 66, no. 3, pp. 272-277, 2016.

[8] K. Guney and S. Basbug, "Null synthesis of time-modulated circular antenna arrays using an improved differential evolution algorithm," IEEE Antennas and Wireless Propagation Letters, vol. 12, pp. 817-820, 2013.

[9] K. Guney, A. Durmus, and S. Basbug, "Antenna array synthesis and failure correction using differential search algorithm," International Journal of Antennas and Propagation, vol. 2014, Article ID 250841, 8 pages, 2014.

[10] S. Mirjalili, S. M. Mirjalili, and A. Hatamlou, "Multi-verse optimizer: a nature-inspired algorithm for global optimization," Neural Computing and Applications, vol. 27, no. 2, pp. 495-513, 2016.

[11] X. Li, K. M. Luk, and B. Duan, "Multiobjective optimal antenna synthesis for microwave wireless power transmission," IEEE Transactions on Antennas and Propagation, vol. 67, no. 4, pp. 2739-2744, 2019.

[12] A. A. Amaireh, A. S. Al-Zoubi, and N. I. Dib, "Sidelobe-level suppression for circular antenna array via new hybrid optimization algorithm based on antlion and grasshopper optimization algorithms," Progress in Electromagnetics Research C, vol. 93, pp. 49-63, 2019.

[13] A. Sharaqa and N. I. Dib, "On the optimal design of nonuniform circular antenna arrays," Journal of Applied Electromagnetism, vol. 14, no. 1, pp. 42-59, 2012.

[14] M. A. Panduro, A. L. Mendez, R. Dominguez, and G. Romero, "Design of non-uniform circular antenna arrays for side lobe reduction using the method of genetic algorithms," $A E U$ International Journal of Electronics and Communications, vol. 60, no. 10, pp. 713-717, 2006.

[15] M. Shibab, Y. Najjar, N. Dib, and M. Khodier, "Design of nonuniform circular antenna arrays using particle swarm optimization," Journal of Electrical Engineering, vol. 59, no. 4, pp. 216-220, 2008.

[16] A. Sharaqa and N. Dib, "Circular antenna array synthesis using firefly algorithm," International Journal of RF and Microwave Computer-Aided Engineering, vol. 24, no. 2, pp. 139-146, 2014.
[17] K. Guney and S. Basbug, "A parallel implementation of seeker optimization algorithm for designing circular and concentric circular antenna arrays," Applied Soft Computing, vol. 22, pp. 287-296, 2014.

[18] M. Rattan, M. S. Patterh, and B. S. Sohi, "Optimization of circular antenna arrays of isotropic radiators using simulated annealing," International Journal of Microwave and Wireless Technologies, vol. 1, no. 5, pp. 441-446, 2009.

[19] G. F. R. Ellis, "Does the multiverse really exist?" Scientific American, vol. 305, no. 2, pp. 38-43, 2011.

[20] M. Tegmark, "Parallel universes," in Science and Ultimate Reality: From Quantum To Cosmos, Cambridge University Press, Cambridge, UK, 2004.

[21] D. M. Eardley, "Death of white holes in the early universe," Physical Review Letters, vol. 33, no. 7, pp. 442-444, 1974

[22] P. J. Steinhardt and N. Turok, "A cyclic model of the universe," Science, vol. 296, no. 5572, pp. 1436-1439, 2002.

[23] P. C. W. Davies, "Thermodynamics of black holes," Reports on Progress in Physics, vol. 41, no. 8, pp. 1313-1355, 1978.

[24] S.-L. Ning and W.-B. Liu, "Black hole phase transition in massive gravity," International Journal of Theoretical Physics, vol. 55, no. 7, pp. 3251-3259, 2016.

[25] M. S. Morris and K. S. Thorne, "Wormholes in spacetime and their use for interstellar travel: a tool for teaching general relativity," American Journal of Physics, vol. 56, no. 5, pp. 395-412, 1988.

[26] D. H. Wolpert and W. G. Macready, "No free lunch theorems for optimization," IEEE Transactions on Evolutionary Computation, vol. 1, no. 1, pp. 67-82, 1997. 